our knowledge of their complexity in vivo is only partial. Here we wished to characterise plaque macrophages from two of the most common murine models; $\mathrm{ApoE}^{-/-}$(Apolipoprotein $\mathrm{E}$ null) and $\mathrm{LDLR}^{-/}$(low density lipoprotein receptor null) and a relatively new and less characterised model; PCKS9AAV8 (Adeno-associated virus serotype 8-proprotein convertase subtilisin kexin type 9) induced hyperlipidaemia.

Using immunohistochemistry, plaques from high-fat diet fed $\mathrm{ApoE}^{-/-}, \mathrm{LDLR}^{-/-}$and PCSK9-AAV8 mice were simultaneously stained for the pan macrophage marker Mac-3 and the pro-/ anti- inflammatory markers inducible nitric oxide synthase (iNOS) and Arginase I (ArgI). Plaques were imaged using fluorescence microscopy and analysed by ImageJ. Individual Mac-3 + cells were selected as the region of interest and corresponding iNOS and ArgI staining was quantified. The analysis allowed consideration for the spectrum of marker co-expression and characterisation of individual cells based on staining intensity. Using this approach, complex populations of plaque macrophagesincluding single+ ArgI, double positive, double negative and single + iNOS were quantified. To understand the roles of these populations in atherosclerosis further, we correlated macrophage quality and quantities with lesion size and collagen content.

We show that $\mathrm{ApoE}^{-/-}$plaque macrophages are significantly more pro-inflammatory than $\mathrm{LDLR}^{-/}$and PCSK9 plaque macrophages $(\mathrm{p}<0.05$ and $\mathrm{p}<0.0001)$. The population responsible for the pro-inflammatory phenotype of $\mathrm{ApoE}^{-/-}$ macrophages were single + iNOS cells $(p<0.0001)$. We also show that the abundance of these cells significantly correlates $\left(\mathrm{R}^{2}=0.4791, \mathrm{p}=0.0183\right)$ with lesion size in the aortic sinus. In addition, the frequency of double negative macrophages correlated with lesion collagen content $\left(R^{2}=0.4451, p=\right.$ 0.0178).

For the first time, plaque macrophages from three murine atherosclerosis models have been comprehensively characterised using a multi-colour image analysis strategy and suggest that plaque macrophages from $\mathrm{ApoE}^{-/-}$mice are significantly more pro-inflammatory than $\mathrm{LDLR}^{-/}$and PCSK9 macrophages. We show that single iNOS+ cells may have a role in promoting lesion formation and double negative cells may also have a role in lesion stability. We envisage our platform provides a novel tool to gain a further, in-depth understanding of macrophage phenotype in atherosclerosis and will use it to elucidate the action of modulators of macrophage polarisation in vivo.

\section{HDAC3 UNCONVENTIONAL SPLICING MEDIATES ENDOTHELIAL-MESENCHYMAL TRANSITION IN CARDIAC FIBROSIS}

Ka Hou Lao*, Dario Ummarino, Ajay Shah, Lingfang Zeng. King's College London; *Presenting Author

\subsection{6/heartjnl-2016-309890.166}

Introduction Endothelial-mesenchymal transition (EndMT) is a process where endothelial cells (ECs) undergo dramatic cytoskeletal remodelling and gene expression changes, leading to a more motile mesenchymal phenotype that can significantly contribute to cardiac fibrosis. Histone deacetylase 3 (HDAC3), a class I HDAC, is essential in the maintenance of endothelial homeostasis. Our previous study indicates that mouse HDAC3 mRNA can undergo unconventional splicing, and that the HDAC3-alpha (HDAC3a) spliced isoform promotes EndMT in human aortic endothelial cells (HAECs). This project aims to elucidate the role of HDAC3a in mediating EndMT and its underlying mechanisms in the development of pressure-overload-induced cardiac fibrosis.

Methods and results Our previous study reveals that HDAC3a promotes EndMT in HAECs via activation of transforming growth factor-beta 2, which could possibly be mediated through increased protease enzyme activity. Therefore HAECs overexpressed with HDAC3a via adenoviral gene transfer (AdHDAC3a) have undergone a protease array, which revealed that cells overexpressed with HDAC3a have an elevated level of a disintegrin and metalloproteinase with thrombospondin motifs 1 (ADAMTS1). This was further confirmed with increased mRNA and protein expression levels of ADAMTS1 in Ad-HDAC3a-infected HAECs using RT-qPCR and Western blotting analysis respectively (both $\mathrm{n}=4, P<0.05$ ). A mouse model of pressure overload-induced cardiac fibrosis was established by transverse aortic constriction (TAC), and cardiac fibrosis was confirmed by increased Col1a1 expression seen using RT-qPCR and Picrosirius red staining in heart tissue sections from mice $7 \mathrm{~d}$ post-TAC. Immunofluorescent staining detected $\mathrm{CD}^{+} 1^{+} /$alpha-SMA ${ }^{+}$(indicative of EndMT) and HDAC $3 \mathrm{a}^{+} / \mathrm{CD} 31^{+}$cells in heart tissues from TAC mice but not in those from sham-operated mice. There were significant increases in mRNA expression of HDAC3a and ADAMTS1, together with EndMT transcription factors Snai1, Snai2 and Twist 1 in heart tissues from $7 \mathrm{~d}$ post-TAC mice compared to those from $7 \mathrm{~d}$ post-sham mice ( $\mathrm{n}=7--8, P<0.05)$. Elevated HDAC3a and ADAMTS1 protein expression were also detected in the TAC heart tissues $(\mathrm{n}=5, P<0.05)$.

Conclusion These results indicate an association between the HDAC3 splicing isoform HDAC3a, ADAMTS1 and EndMT during TAC-induced cardiac fibrosis. Further investigation of the underlying mechanisms mediated by HDAC3a and ADAMTS1 in cardiac fibrosis is warranted.

\section{THE MECHANISM OF P22PHOX C242T SNP INHIBITION OF TNF ALPHA-INDUCED NOX2 ACTIVATION IN HUMAN ENDOTHELIAL CELLS AND VESSELS}

${ }^{1}$ Daniel Meijles*, ${ }^{2}$ Lampson Fan, ${ }^{3}$ Maziah Ghazaly, ${ }^{1}$ Jian-Mei Li. ' University of Reading; ${ }^{2}$ Royal Berkshire Hospital; ${ }^{3}$ University of Surrey; ${ }^{*}$ Presenting Author

10.1136/heartjnl-2016-309890.167

NADPH oxidase, by generating reactive oxygen species, is involved in the pathophysiology of many cardiovascular diseases and represents a therapeutic target for the development of novel drugs. A single-nucleotide polymorphism (SNP) C242T of the $\mathrm{p} 22^{\text {phox }}$ subunit of NADPH oxidase has been reported to be negatively associated with coronary heart disease (CHD) and may predict disease prevalence. However, the underlying mechanisms remain unknown.

Using computer molecular modelling we discovered that C242T SNP causes significant structural changes in the extracellular loop of $\mathrm{p} 22^{\text {phox }}$ and reduces its interaction stability with the catalytic Nox2 subunit. Gene transfection of human pulmonary microvascular endothelial cells showed that C242T p $22^{\text {phox }}$ reduced significantly Nox 2 expression, but had no significant effect on basal endothelial superoxide $\left(\mathrm{O}_{2}{ }^{-}\right)$production, or the expression of Nox1 and Nox4. When cells were stimulated with TNFalpha (or high glucose), C242T $\mathrm{p} 22^{\text {phox }}$ inhibited significantly TNFalpha-induced Nox2 maturation, 
$\mathrm{O}_{2}{ }^{--}$production, MAPK and $\mathrm{NFkB}$ activation, and monocyte adhesion (all $\mathrm{p}<0.05$; one-way AVOVA). These C242T effects were further confirmed using $\mathrm{p} 22^{\text {phox }}$ shRNA engineered HeLa cells and coronary microvascular endothelial cells isolated from Nox2 knockout mice. Clinical significance was investigated using saphenous vein segments from non-CHD subjects after phlebectomies. Informed consents were obtained from the patients and the project was approved by the local NHS and the university ethical committees according to the UK regulation. TT $(\mathrm{C} 242 \mathrm{~T})$ allele was common (prevalence of $20 \%$ ) and compared to CC, veins bearing TT allele had significantly lower levels of Nox2 expression and $\mathrm{O}_{2}{ }^{--}$generation in response to high glucose challenge (all p < 0.05).

In summary, our study for the first time provides mechanistic insight into the protective effect of the $\mathrm{p} 22^{\text {phox }} \mathrm{C} 242 \mathrm{~T}$ SNP against the inflammatory oxidative stress-related cardiovascular diseases. $\mathrm{p} 22^{\text {phox }} \mathrm{C} 242 \mathrm{~T}$ SNP causes $\mathrm{p} 22^{\text {phox }}$ structural changes that alter its interaction with the catalytic subunit Nox2 and inhibits endothelial oxidative response to TNFalpha or high glucose stimulation.

\section{TARGETING THE HIPPO SIGNALLING PATHWAY TO ENHANCE THE THERAPEUTIC POTENTIAL OF IPS- DERIVED CARDIOMYOCYTES}

Abigail Robertson*, Elizabeth J Cartwright, Delvac Oceandy. University of Manchester; *Presenting Author

\subsection{6/heartjnl-2016-309890.168}

Introduction Cell based therapy using stem cell derived cardiomyocytes, has emerged as a potential therapeutic approach for cardiac diseases such as myocardial infarction and heart failure. Adult skin fibroblasts can be reprogrammed into induced pluripotent stem cells (iPSC) which could be an ideal source of iPS-derived cardiomyocytes (iPS-CM). Challenges facing cell therapy include the high number of viable cells needed to survive in pathological conditions. The Hippo signalling pathway has been described as a key pathway involved in regulating cardiomyocyte proliferation and survival in both embryonic and adult hearts. The purpose of this study is to test whether modification of the Hippo pathway will enhance the efficiency of iPS-CM generation and will increase iPS-CM survival and viability in pathological conditions.

Methods Skin fibroblasts were reprogrammed to iPS cells and then differentiated to cardiomyocytes. The Hippo signalling pathway was modified by genetic ablation of MST1, a major upstream regulator of the Hippo pathway, or by overexpressing YAP, the main downstream effector of the pathway. Cell proliferation was analysed using EdU incorporation assay and staining for cytokinesis markers Ki67 and phospho-histone H3. Cell death and viability were analysed using caspase $3 / 7$ and MTT activity and trypan blue staining in both normal and hypoxic conditions.

Results Analysis of cell proliferation shows that genetic ablation of Mst1 leads to significantly increased proliferation (12 $\pm 1.5 \% \mathrm{P}<0.001)$, survival and viability $(20 \pm 4.3 \% \mathrm{P}<$ 0.001 ) of iPSC in both normal and hypoxic conditions compared to controls. In addition overexpression of YAP, which is normally inhibited by upstream Hippo pathway components, and overexpression of mutated constitutively active form of YAP (S127A) increases cell proliferation in iPS-CM compared to control iPS-CM as shown with EdU assay $(+20.8 \pm 1.6 \%$
$\mathrm{P}<0.01)$ and Ki67 staining $(4.9 \pm 0.9 \% \mathrm{P}<0.001)$. Overexpression of YAP leads to up regulation of genes associated with inhibition of apoptosis and promotion of cell proliferation.

Conclusion Targeting the Hippo pathway in iPS cells and iPS$\mathrm{CM}$ significantly increases proliferation and survival in both normal and hypoxic conditions. Therefore, modulation of the Hippo pathway could become a new strategy to enhance the therapeutic potential of iPS-CM.

\section{SOLUBLE GUANYLATE CYCLASE ACTIVATORS AS COMBINATION ANTI-PLATELET THERAPY WITH P2Y12 INHIBITORS AND PDE INHIBITORS: IN VIVO AND EX VIVO STUDIES}

${ }^{1}$ Plinio Ferreira*, ${ }^{2}$ Paul Armstrong, ${ }^{2}$ Melissa Chan, ${ }^{2}$ Timothy Warner. ${ }^{1}$ University of SÃfO Paulo/William Harvey Research Institute; ${ }^{2}$ William Harvey Research Institute; *Presenting Author

10.1136/heartjnl-2016-309890.169

Methods Mice were pre-treated with vehicle, prasugrel (0.3 $\mathrm{mg} / \mathrm{kg})$, cinaciguat $(0.3 \mathrm{mg} / \mathrm{kg})+$ dipyridamole $(2.0 \mathrm{mg} / \mathrm{kg})$, or prasugrel + cinaciguat + dipyridamole (triple) and anesthetized. To measure in vivo thrombus formation, the carotid artery was then exposed and thrombosis induced by placement of a piece of filter paper saturated with $10 \%$ ferric chloride in contact with the adventitial surface of vessel for 3 minutes. Carotid artery blood flow was monitored by a Doppler probe with the time to form an occlusive thrombus being taken as the time required for blood to stop flowing completely for $>1$ minutes. Time to occlusion from different treatment groups was compared. To measure ex vivo platelet function, blood was taken from the vena cava of treated mice and aggregation of platelets in whole blood in response to arachidonic acid (AA) $1 \mathrm{mM}$, PAR-4 amide $30 \mu \mathrm{M}$, and collagen 10 $\mu \mathrm{g} / \mathrm{ml}$ was then determined using flow cytometry.

Results In mice treated with vehicle, prasugrel and cinaciguat + dipyridamole complete vessel occlusion occurred within 8 minutes. Conversely, triple combination of prasugrel + cinaciguat + dipyridamole blocked thrombus formation (time to occlusion $>24$ minutes). In ex vivo platelet function tests, we observed reduced platelet aggregation in mice treated with the triple combination compared to other treatments. Results as mean \pm SEM. AA; vehicle $83 \pm 9 \%$, prasugrel $67 \pm 7 \%$, cinaciguat + dipyridamole $62 \pm 9 \%$, triple $27 \pm 27 \%$ : PAR-

Prasugrel $(0.3 \mathrm{mg} / \mathrm{kg}) \&$ Dipyridamole $(2 \mathrm{mg} / \mathrm{kg}) \&$ Cinaciguat $(0.3 \mathrm{mg} / \mathrm{kg})$

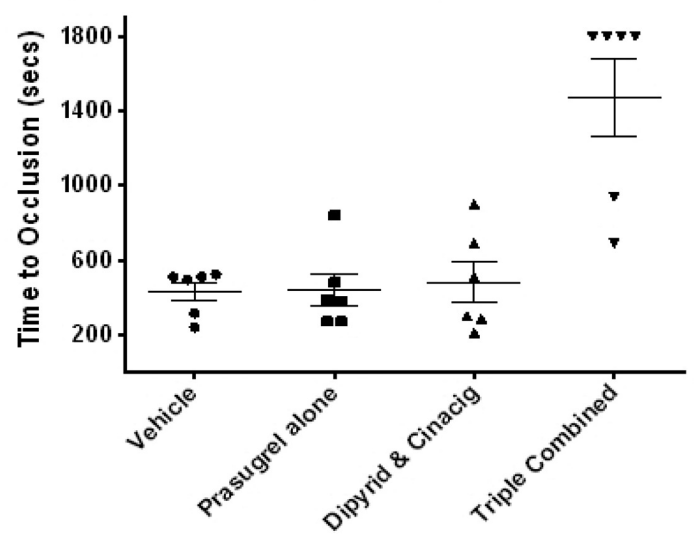

Abstract 169 Figure 1 